\title{
The prevalence of chronic fatigue syndrome/ myalgic encephalomyelitis: a meta-analysis
}

This article was published in the following Dove Press journal:

Clinical Epidemiology

25 March 2013

Number of times this article has been viewed

\author{
Samantha Johnston' \\ Ekua W Brenu' \\ Donald Staines ${ }^{1,2}$ \\ Sonya Marshall-Gradisnik' \\ 'Griffith Health Institute, School \\ of Medical Sciences, National \\ Centre for Neuroimmunology and \\ Emerging Diseases, Griffith University, \\ Parklands, QLD, Australia; ${ }^{2}$ Gold \\ Coast Public Health Unit, Queensland \\ Health, Robina, QLD, Australia
}

Purpose: To perform a meta-analysis to examine variability among prevalence estimates for CFS/ME, according to the method of assessment used.

Methods: Databases were systematically searched for studies on CFS/ME prevalence in adults that applied the 1994 Centers for Disease Control (CDC) case definition. ${ }^{1}$ Estimates were categorized into two methods of assessment: self-reporting of symptoms versus clinical assessment of symptoms. Meta-analysis was performed to pool prevalences by assessment using random effects modeling. This was stratified by sample setting (community or primary care) and heterogeneity was examined using the $I^{2}$ statistic.

Results: Of 216 records found, 14 studies were considered suitable for inclusion. The pooled prevalence for self-reporting assessment was 3.28\% (95\% CI: $2.24-4.33$ ) and $0.76 \%$ (95\% CI: 0.23-1.29) for clinical assessment. High variability was observed among self-reported estimates, while clinically assessed estimates showed greater consistency.

Conclusion: The observed heterogeneity in CFS/ME prevalence may be due to differences in method of assessment. Stakeholders should be cautious of prevalence determined by the selfreporting of symptoms alone. The 1994 CDC case definition appeared to be the most reliable clinical assessment tool available at the time of these studies. Improving clinical case definitions and their adoption internationally will enable better comparisons of findings and inform health systems about the true burden of CFS/ME.

Keywords: chronic fatigue syndrome, myalgic encephalomyelitis, prevalence, meta-analysis

\section{Introduction}

Chronic fatigue syndrome or myalgic encephalomyelitis (CFS/ME) is most commonly characterized by fatigue lasting more than 6 months accompanied by symptoms such as muscle and joint pain, sore throat, tender lymph nodes, and cognitive difficulties. ${ }^{1}$ It is not relieved by rest and results in a substantial reduction in the patient's activity levels prior to onset.

Studies on the prevalence of this condition have been available since 1990. While most reports have come from the United States and Europe, increasing estimates are emerging from Asia and developing countries, such as Nigeria. ${ }^{2-5}$ Prevalence varies from as low as $0.2 \%$ to as high as $6.41 \% .{ }^{6,7} \mathrm{~A}$ previous review suggested that the inconsistency is more likely due to differences in study design rather than true differences in prevalence. ${ }^{8}$ Prior to epidemiological surveys, prevalence was suggested based on clinical reviews of patients in tertiary care. ${ }^{9}$ The first studies to use prospective sampling methods were based on physician referrals. ${ }^{10-13}$ Studies gradually began to directly screen samples from primary care clinics, ${ }^{13-18}$ and the wider community, ${ }^{2,4-6,19-28}$ 
through questionnaires and structured interviews. In contrast, larger population based studies first screen medical databases for potential cases. ${ }^{7,16}$

A particular issue in the development of prevalence studies is case definitions with fundamental differences with respect to inclusion criteria for comorbid and psychiatric conditions. Several studies have demonstrated the difference in prevalence detected according to the case definition used. ${ }^{2,7,14,17,23}$ In Iceland, for example, prevalence was estimated as $4.8 \%, 2.4 \%$, and $1.4 \%$ using the Australian, Oxford, and 1994 CDC criteria, respectively. ${ }^{23}$ Even when the same case definition is applied across studies, different methods have been used to ascertain cases. Many studies rely on the self-reporting of symptoms alone, ${ }^{2,4,6,15,19,21,23,25,27}$ while others rely on complete clinical assessment of symptoms. ${ }^{3,5,7,14,17,20,22,24,26}$ However, the effect of study design on prevalence has not been examined.

This paper presents the findings of a meta-analysis performed to assess the consistency between estimates. The aim was to verify whether prevalence varied according to method of assessment used to detect cases. It was hypothesized that prevalence estimates relying on the self-reporting of symptoms would, on average, be higher and less consistent than estimates based on clinical assessment. The assessment was completed using the guidelines of the Meta-analysis for Observational Studies in Epidemiology (MOOSE) Group. ${ }^{29}$

\section{Method}

\section{Literature search}

Systematic searches of the Medline, Embase, and Pubmed Central databases were conducted using the Medical Search Headings (MeSH terms) "Chronic Fatigue Syndrome” (which also captures myalgic encephalomyelitis) and "prevalence." No limit was applied to years published or language. The strategy also included a secondary search of reference lists of records retrieved from the databases.

\section{Selection of studies}

Titles and abstracts were screened for potential studies and full text articles were assessed for suitability. The outcome of interest was prospective studies on the point prevalence of CFS/ $\mathrm{ME}$, as defined by the authors of each study. Period prevalence was not considered as it could have resulted in inflated prevalence estimates when compared to point prevalence. Selected studies were based on community or primary care samples, where the condition is most often presented.

Studies on secondary and tertiary care patients were excluded as high-risk groups, as were groups of special interest that did not represent the general population, such as veterans and nurses.

Studies published in languages other than English were also included if detailed English summaries were available. Only studies that applied the 1994 CDC case definition were selected. This was identified as the most widely applied criteria among prevalence studies. This case definition was the most widely accepted definition available at the time of these studies, is also the current criteria used by the CDC, and is more selective than the previously proposed Australian ${ }^{10}$ and Oxford criteria. ${ }^{30}$ Furthermore, only studies on individuals aged 18 years and older were included as the 1994 CDC definition was designed for the detection of CFS/ME in adults. ${ }^{1}$

\section{Data extraction and analysis}

Data on sample size, response rate, number of cases detected, method of assessment (self-reported versus clinical assessment), and sample setting (community versus primary care) were extracted. Sample size was calculated as the total number of participants invited to the study minus the number of non-responders. Prevalence was tabulated as the number of cases detected divided by the sample size, along with standard errors. All estimates were expressed as percentage of the population. Separate tabulations were made according to method of assessment, sample setting, age, and gender. The inverse variance method by DerSimonian and Laird, ${ }^{31}$ adjusted for random effects, was used to calculate pooled prevalence and 95\% confidence intervals (CI) for self-reported and clinically assessed symptoms of CFS/ME. Heterogeneity between studies was tested using the $I^{2}$ statistic. Sensitivity analysis was performed to test the influence of possible outliers. The meta-analysis was performed in STATA v.10.0. Studies that reported prevalence for more than one study site or for both methods of assessment were treated as separate studies for the purpose of modeling.

\section{Results}

The literature search found 218 records, including 26 prevalence studies that were further assessed for eligibility (Figure 1). Of these, 11 exclusions were made: 10 did not use the 1994 CDC case definition for CFS/ME ${ }^{10-16,19,20,28}$ (including three reporting period prevalence ${ }^{12,13,16}$ ); and one study recruited participants with acute viral illness as part of a case control design. ${ }^{17}$ During sensitivity analysis, a further study ${ }^{7}$ with a statistical weight of more than $90 \%$ was excluded from the investigation.

Fourteen studies, published between 1995 and 2011, were considered suitable for meta-analysis. Eleven were based on 


\begin{tabular}{|l|}
\hline Search strategy \\
Computer search \\
Medline (1950- \\
Aug 2012) MeSH \\
Heading = (fatigue \\
syndrome chronic) AND \\
MeSH \\
Heading: exp = \\
(prevalence) \\
Embase (1966- \\
Aug 2012) 'chronic \\
fatigue \\
syndrome'/exp/mj AND \\
'prevalence'/exp/mj \\
AND [embase]/lim \\
PubMed central (1951- \\
Aug 2012) (chronic \\
fatigue syndrome \\
[MeSH terms]) AND \\
prevalence [MeSH \\
terms] \\
Reference list search \\
\end{tabular}

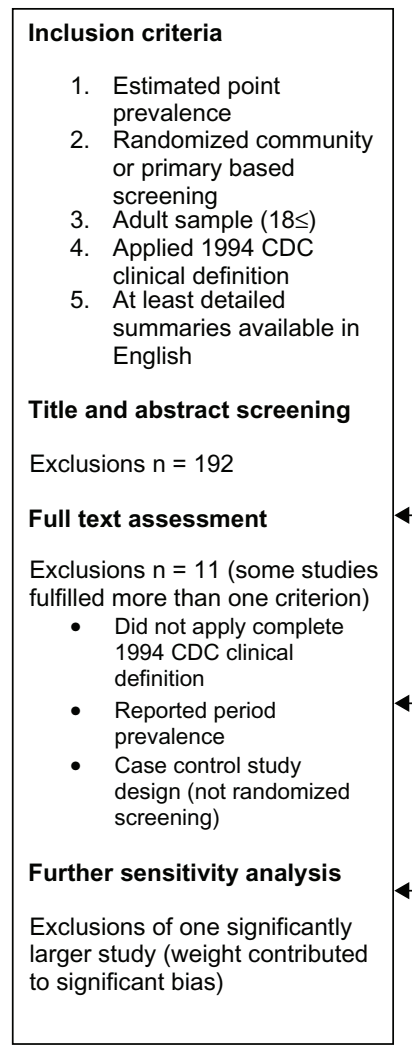

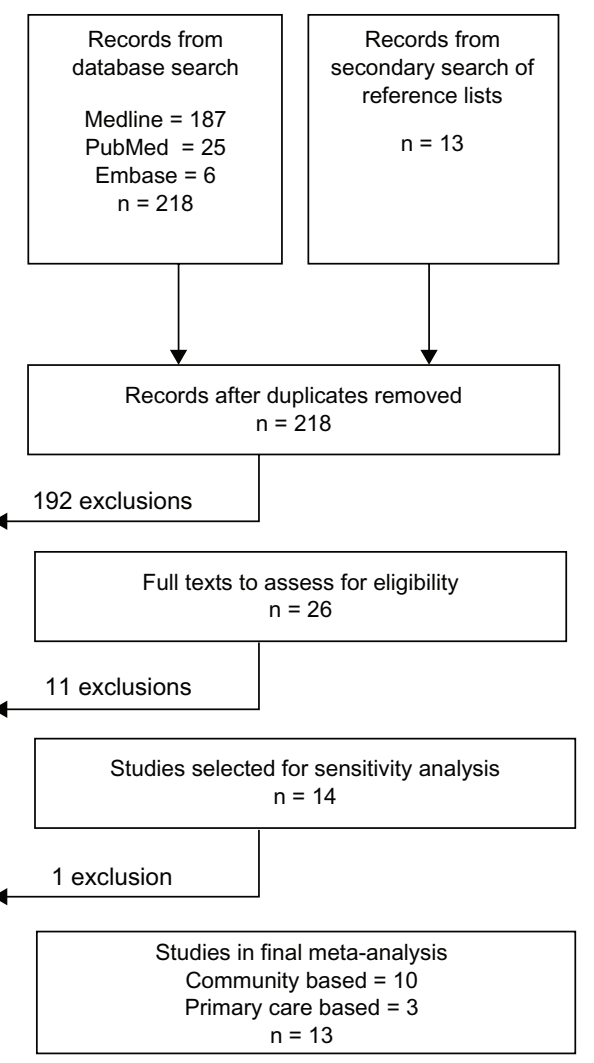

Figure I Flow chart for the selection of prevalence studies for the meta-analysis.

community samples and three were based on primary care samples. Most studies reported CFS/ME cases based on the self-reporting of symptoms alone. , $, 6,20,21,23,25,27$ Three studies reported cases after clinical assessment of symptoms, $, 3,5,18$ while four studies provided estimates for both methods. ${ }^{4,22,24,26}$ Including one study that contributed estimates for two separate study sites (UK and Brazil), a total of 19 estimates were tested by meta-analysis. Insufficient data were found in more than $50 \%$ of the studies, thereby preventing the calculation of summaries of age-gender specific prevalence.

The overall, pooled prevalence for self-reported CFS/ME was 3.48\% (95\% CI: 2.36-4.60) and high heterogeneity was observed $\left(I^{2}=58 \%\right)$. All samples were community-based. The overall, pooled prevalence of $\mathrm{CFS} / \mathrm{ME}$ detected with clinical assessment was low at $0.76 \%$ (95\% CI: $0.23-1.29)$ and no heterogeneity was detected $\left(I^{2}=0 \%\right)$. Heterogeneity remained

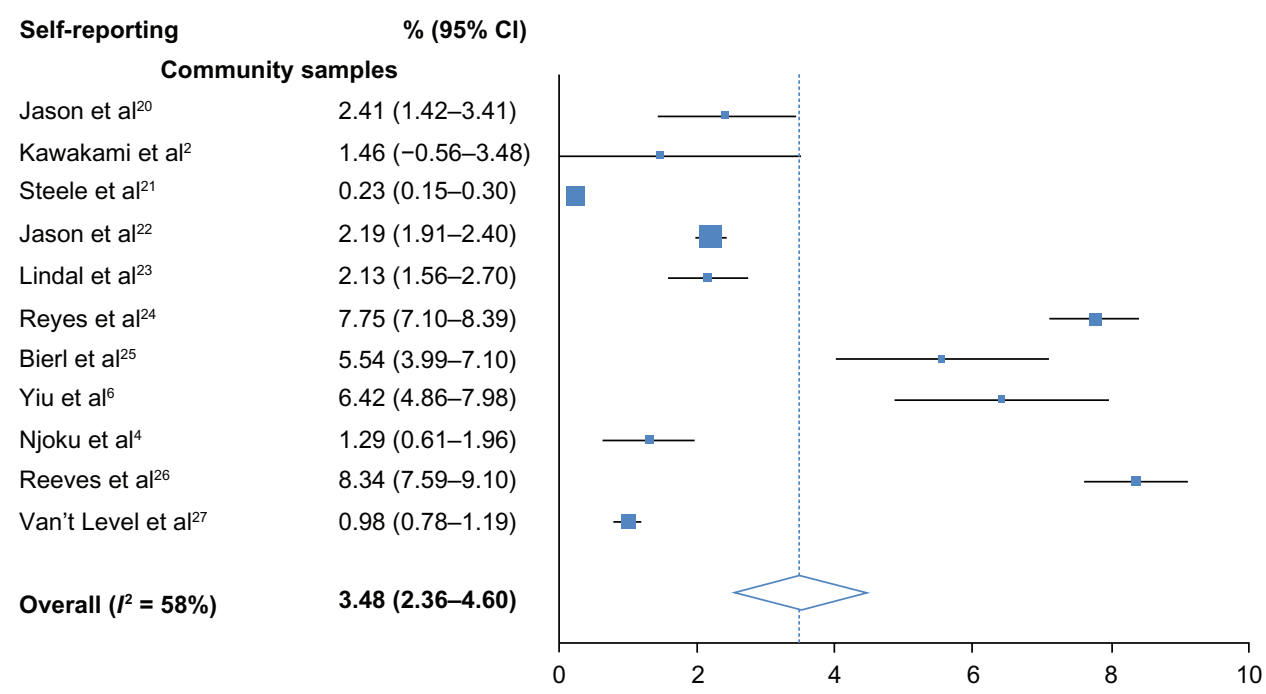

Figure 2 Prevalence of CFS/ME detected by self-reporting. Abbreviation: $\mathrm{Cl}$, confidence interval. 


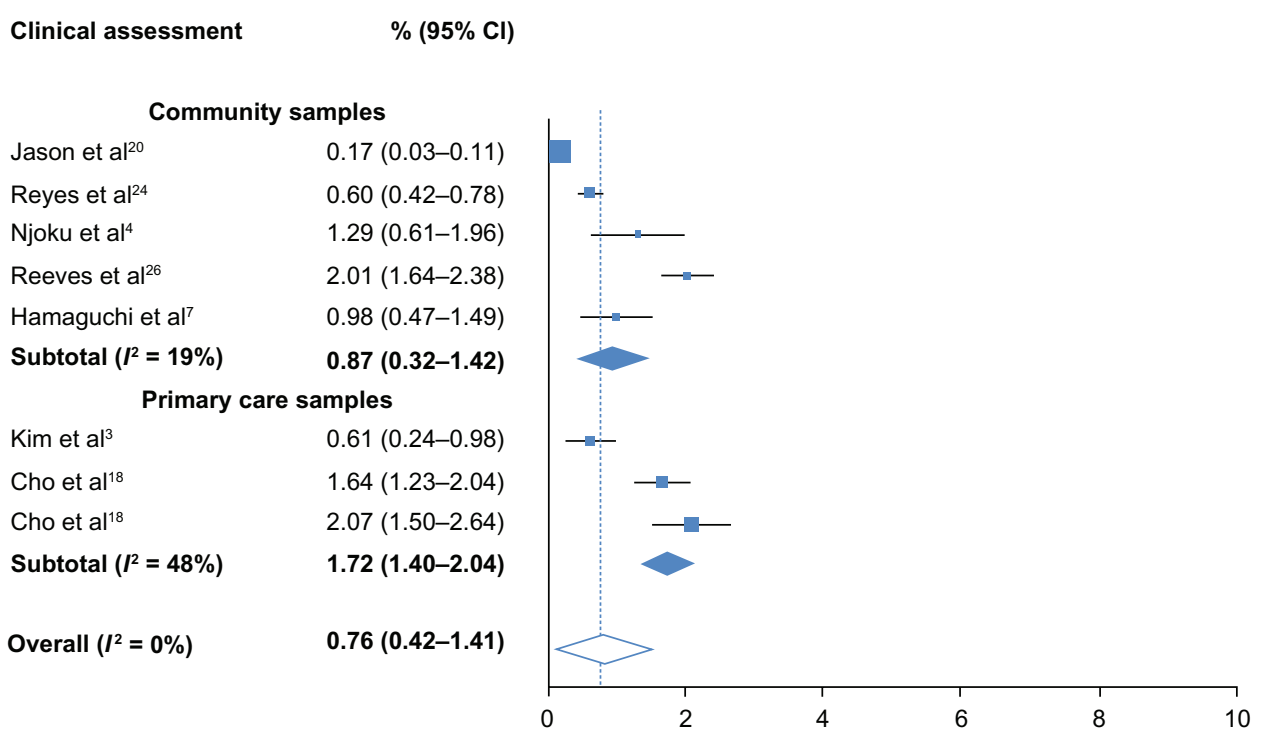

Figure 3 Prevalence of CFS/ME detected by clinical assessment. Abbreviation: $\mathrm{Cl}$, confidence interval.

lower than self-reporting studies when estimates were systematically removed during sensitivity analysis. Prevalence however, was lower in community samples $(0.87 \%$; $0.32-1.42)$ than in primary care samples $(1.72 \% ; 1.40-2.04)$. Low heterogeneity $\left(I^{2}=19 \%\right)$ was found among community samples. Moderate heterogeneity was detected between the three primary care samples $\left(I^{2}=48 \%\right)$.

\section{Discussion}

This review demonstrated that high heterogeneity is found among prevalence estimates that rely on the self-reporting of symptoms. This determination was based on community samples only, as available estimates from primary care samples were not eligible for this meta-analysis. Homogeneity, however, was found between studies that completed clinical assessment of symptoms. Furthermore, the findings illustrate that prevalence estimates obtained from self-reporting alone are higher than estimates involving clinical assessment.

Those attending primary care clinics may be a higher risk group than those in the general community. Slightly higher prevalence was found in primary care, but this was more likely due to the limited availability of studies. This also made the heterogeneity detected among primary care samples highly sensitive to the lower prevalence detected in a Korean sample. ${ }^{3}$ However, the variability among community samples that used clinical assessment was still low compared to community samples relying on self-reporting.

This systematic review used specific inclusion criteria to minimize a biased selection of studies. The majority of exclusions were studies based on dated definitions of CFS/ME. A UK study ${ }^{7}$ based on nationwide screening was also removed due to its large statistical weighting. If included, the results of the remaining studies would not have been detected by the meta-analysis. Prevalences were adjusted for no response or participation. This may have resulted in higher estimates, as it assumes that non-responders are not less likely to have CFS/ME.

Although there are studies that rely on self-reporting to determine the official prevalence of CFS/ME, 2,6,23,27 many use it as an initial screening technique to source potential cases of CFS/ME and assess the feasibility of conducting larger epidemiological surveys. In such cases, those that report symptoms fulfilling the clinical definition of CFS/ME have often been referred to as CFS/ME-like cases. ${ }^{21,24-27}$ It is not uncommon for studies to apply further tools to help verify suspicions of CFS/ME, such as empirical criteria, ${ }^{32}$ validated health surveys, ${ }^{33}$ fatigue scores, ${ }^{34}$ and depression scales. ${ }^{35}$ Some studies have then proceeded with clinical diagnosis of CFS/ME. Different approaches to clinical assessment can be found; one study evaluated all participants as part of a random health check of the population. ${ }^{5}$ Some studies evaluated those reporting CFS/ME symptoms, ${ }^{18,36}$ while others only evaluated a sample reporting CFS/ME symptoms..$^{4,22,24,26}$ The latter may have resulted in conservative estimates as cases may have been detected in those not assessed.

The differences found in heterogeneity due to method of assessment highlight the need for collaborative research in $\mathrm{CFS} / \mathrm{ME}$ prevalence where similar methods are applied across study sites. This has only been demonstrated by one study that found similar prevalences of CFS/ME in Brazil 
$(1.64 \%$; $95 \%$ CI: $1.23-2.04)$ and the UK $(2.07 \% ; 1.50-2.64) .{ }^{18}$ The current meta-analysis particularly illustrates that prevalence is more consistent across samples when clinical assessment is involved. Therefore, it is recommended that studies combine the use of a standard case definition with clinical verification of symptoms. More specific definitions are now available, such as the recently released International Consensus definition. ${ }^{32}$ Their use to assess prevalence should also help produce more reliable estimates in the future.

\section{Conclusion}

Prevalence estimates for CFS/ME based on self-reporting alone should be viewed with caution. Clinically valid diagnoses are vital in undertaking accurate prevalence studies for CFS/ME. The findings of this study are based on CFS/ME as defined by the CDC. As new advances are made in clinical case definitions, for example, through the International Consensus definition, further valid prevalence studies may be expected.

\section{Disclosure}

The authors report no conflicts of interest in this work.

\section{References}

1. Fukuda K, Straus SE, Hickie I, Sharpe MC, Dobbins JG, Komaroff A. The chronic fatigue syndrome: a comprehensive approach to its definition and study. International Chronic Fatigue Syndrome Study Group. Ann Intern Med. 1994;121(12):953-959.

2. Kawakami N, Iwata N, Fujihara S, Kitamura T. Prevalence of chronic fatigue syndrome in a community population in Japan. Tohoku J Exp Med. 1998;186(1):33-41.

3. Kim CH, Shin HC, Won CW. Prevalence of chronic fatigue and chronic fatigue syndrome in Korea: community-based primary care study. J Korean Med Sci. 2005;20(4):529-534.

4. Njoku MG, Jason LA, Torres-Harding SR. The prevalence of chronic fatigue syndrome in Nigeria. J Health Psychol. 2007;12(3):461-474.

5. Hamaguchi M, Kawahito Y, Takeda N, Kato T, Kojima T. Characteristics of chronic fatigue syndrome in a Japanese community population: chronic fatigue syndrome in Japan. Clin Rheumatol. 2011;30(7):895-906.

6. Yiu YM, Qiu MY. A preliminary epidemiological study and discussion on traditional Chinese medicine pathogenesis of chronic fatigue syndrome in Hong Kong. Zhong Xi Yi Jie He Xue Bao. 2005;3(5): 359-362. Chinese.

7. Nacul LC, Lacerda EM, Pheby D, et al. Prevalence of myalgic encephalomyelitis/chronic fatigue syndrome (ME/CFS) in three regions of England: a repeated cross-sectional study in primary care. BMC Med. 2011;9:91.

8. Ranjith G. Epidemiology of chronic fatigue syndrome. Occup Med (Lond). 2005;55(1):13-19.

9. Murdoch JC. Myalgic encephalomyelitis (ME) syndrome-an analysis of the clinical findings in 200 cases. N Z Fam Physician. 1987;14:51-54.

10. Lloyd AR, Hickie I, Boughton CR, Spencer O, Wakefield D. Prevalence of chronic fatigue syndrome in an Australian population. Med J Aust. 1990;153(9):522-528.

11. Ho-Yen DO, McNamara I. General practitioners' experience of the chronic fatigue syndrome. Br J Gen Pract. 1991;41(349):324-326.
12. Gunn WJ, Connell DB, Randall B. Epidemiology of chronic fatigue syndrome: the Centers for Disease Control Study. Ciba Found Symp. 1993;173:83-93; discussion 93-101.

13. Reyes M, Gary HE Jr, Dobbins JG, et al. Surveillance for chronic fatigue syndrome - four US cities, September 1989 through Aug 1993. MMWR CDC Surveill Summ. 1997;46(2):1-13.

14. Bates DW, Schmitt W, Buchwald D, et al. Prevalence of fatigue and chronic fatigue syndrome in a primary care practice. Arch Intern Med. 1993;153(24):2759-2765.

15. Lawrie SM, Pelosi AJ. Chronic fatigue syndrome in the community. Prevalence and associations. Br J Psychiatry. 1995;166(6): 793-797.

16. Versluis RG, de Waal MW, Opmeer C, Petri H, Springer MP. Prevalence of chronic fatigue syndrome in 4 family practices in Leiden. Ned Tijdschr Geneeskd. 1997;141(31):1523-1526. Dutch.

17. Wessely S, Chalder T, Hirsch S, Wallace P, Wright D. The prevalence and morbidity of chronic fatigue and chronic fatigue syndrome: a prospective primary care study. Am J Public Health. 1997;87(9): 1449-1455.

18. Cho HJ, Menezes PR, Hotopf M, Bhugra D, Wessely S. Comparative epidemiology of chronic fatigue syndrome in Brazilian and British primary care: prevalence and recognition. Br J Psychiatry. 2009;194(2): 117-122.

19. Price RK, North CS, Wessely S, Fraser VJ. Estimating the prevalence of chronic fatigue syndrome and associated symptoms in the community. Public Health Rep. 1992;107(5):514-522.

20. Jason LA, Taylor R, Wagner L, et al. Estimating rates of chronic fatigue syndrome from a community-based sample: a pilot study. Am J Community Psychol. 1995;23(4):557-568.

21. Steele L, Dobbins JG, Fukuda K, et al. The epidemiology of chronic fatigue in San Francisco. American J Med. 1998;105(3A):83S-90S.

22. Jason LA, Richman JA, Rademaker AW, et al. A community-based study of chronic fatigue syndrome. Arch Intern Med. 1999;159(18): 2129-2137.

23. Lindal E, Stefansson JG, Bergmann S. The prevalence of chronic fatigue syndrome in Iceland - a national comparison by gender drawing on four different criteria. Nord J Psychiatry. 2002;56(4):273-277.

24. Reyes M, Nisenbaum R, Hoaglin DC, et al. Prevalence and incidence of chronic fatigue syndrome in Wichita, Kansas. Arch Intern Med. 2003;163(13):1530-1536.

25. Bierl C. Regional distribution of fatiguing illnesses in the United States: a pilot study. Popul Health Metr. 2004;2(1):1.

26. Reeves WC, Jones JF, Maloney E, et al. Prevalence of chronic fatigue syndrome in metropolitan, urban, and rural Georgia. Popul Health Metr. 2007;5:5.

27. van't Leven M, Zielhuis GA, van der Meer JW, Verbeek AL, Bleijenberg G. Fatigue and chronic fatigue syndrome-like complaints in the general population. Eur J Public Health. 2010;20(3):251-257.

28. Bhui KS, Dinos S, Ashby D, Nazroo J, Wessely S, White PD. Chronic fatigue syndrome in an ethnically diverse population: the influence of psychosocial adversity and physical inactivity. BMC Med. 2011;9:26.

29. Stroup DF, Berlin JA, Morton SC, et al. Meta-analysis of observational studies in epidemiology: a proposal for reporting. Meta-analysis Of Observational Studies in Epidemiology (MOOSE) group. JAMA. 2000;283(15):2008-2012.

30. Sharpe MC, Archard LC, Banatvala JE, et al. A report - chronic fatigue syndrome: guidelines for research. J R Soc Med. 1991;84(2): $118-121$.

31. DerSimonian R, Laird NM. Meta-anaysis in clinical trials. Control Clin Trials. 1986;7:177-188.

32. Levine PH. Epidemic neuromyasthenia and chronic fatigue syndrome: epidemiological importance of a cluster definition. Clin Infect Dis. 1994;18 Suppl 1:S16-S20.

33. Shea SC, Barney C. Macrotraining: a "how-to" primer for using serial role-playing to train complex clinical interviewing tasks such as suicide assessment. Psychiatr Clin North Am. 2007;30(2):e1-e29. 
34. Colson P, Tamalet C, Raoult D. SVARAP and aSVARAP: simple tools for quantitative analysis of nucleotide and amino acid variability and primer selection for clinical microbiology. BMC Microbiol. 2006;6:21.

35. House K, Ireland AJ, Sherriff M. An investigation into the use of a single component self-etching primer adhesive system for orthodontic bonding: a randomized controlled clinical trial. J Orthod. 2006;33(1):38-44; discussion 28.
36. Kim SH, Lee K, Lim HS. Prevalence of chronic widespread pain and chronic fatigue syndrome in Korean livestock raisers. J Occup Health. 2008;50(6):525-528.

\section{Publish your work in this journal}

Clinical Epidemiology is an international, peer-reviewed, open access journal focusing on disease and drug epidemiology, identification of risk factors and screening procedures to develop optimal preventative initiatives and programs. Specific topics include: diagnosis, prognosis, treatment, screening, prevention, risk factor modification, systematic

Submit your manuscript here: http://www.dovepress.com/clinical-epidemiology-journal
Dovepress

reviews, risk \& safety of medical interventions, epidemiology \& biostatical methods, evaluation of guidelines, translational medicine, health policies \& economic evaluations. The manuscript management system is completely online and includes a very quick and fair peer-review system, which is all easy to use. 